\section{EMPRESAS BIOTECNOLÓGICAS Y DIVULGACIÓN DE LA CIENCIA. EL CASO DE LAS PYMES BIOTECNOLÓGICAS ASENTADAS EN PARQUES TECNOLÓGICOS DE ANDALUCÍA}

\author{
Mạ Luisa García-Hernández \\ Universidad de Málaga \\ mluisagarcia@uma.es \\ Estrella Martínez-Rodrigo \\ Universidad de Granada \\ emrodrigo@ugr.es \\ Juan Salvador Victoria Mas \\ Universidad de Málaga \\ jsvictoria@uma.es
}

\begin{abstract}
Cómo citar este artículo/Citation: García-Hernández, M. L., Martínez-Rodrigo, E. y Victoria Mas, J. S. (2016). Empresas biotecnológicas y divulgación de la ciencia. El caso de las pymes biotecnológicas asentadas en parques tecnológicos de Andalucía. Arbor, 192 (779): a323. doi: http://dx.doi. org/10.3989/arbor.2016.779n3013
\end{abstract}

Recibido: 13 noviembre 2014. Aceptado: 9 marzo 2015

RESUMEN: Las empresas biotecnológicas no pueden desestimar que el interés social por esa franja de conocimiento es muy alto, genera expectativas y recelos, y cubrir esa demanda no solo compete a los organismos públicos. Para comprobar cuál es su realidad enfocamos en este trabajo a las 32 pymes asentadas en cuatro parques tecnológicos de Andalucía. Utilizamos metodologías variadas para comprobar cómo es su presencia en los periódicos locales de referencia en sus respectivas provincias; qué ámbitos cubren sus sitios web y cuál es la opinión de sus gestores sobre diferentes aspectos de su comunicación. Los resultados nos llevan a concluir que no existe diálogo entre la sociedad y estas empresas, paso ineludible para que las pymes integren mecanismos de divulgación como elementos de su comunicación y de su RSC; sin embargo, sus ejecutivos no repudian a la ciudadanía como público, si bien no han localizado las herramientas que solucionen tal desconexión.

PALABRAS CLAVE: Divulgación de la ciencia; pymes biotecnológicas; parques tecnológicos; sociedad; comunicación.
BIOTECHNOLOGICAL COMPANIES AND SPREADING OF SCIENCE. THE CASE OF SMES BASED IN ANDALUSIAN TECHNOLOGICAL PARKS

Copyright: (c) 2016 CSIC. Este es un artículo de acceso abierto distribuido bajo los términos de la licencia Creative Commons Attribution (CC BY) España 3.0.

ABSTRACT: Biotech companies cannot ignore the social interest they arouse in this age of knowledge; this interest raises expectations and fears, and meeting the demand created is not only the responsibility of government agencies. To analyse the situation this paper focuses on 32 SMEs based in four Technology Parks in Andalusia. We use a variety of methods to examine their presence in the local newspapers in their respective provinces; what areas their websites cover and find the opinion of their managers on various aspects of their communications. The results revealed an absence of dialogue between society and these firms. It might be necessary for SMEs to integrate disclosure mechanisms within their communication strategies and their CSR; however, their executives did not repudiate the public as an audience, but have not found the tools to bridge this gap.

KEYWORDS: popular science; biotech SMEs; technology parks; society; communication. 


\section{INTRODUCCIÓN}

El proceso de transición de la sociedad industrial a la sociedad del conocimiento tiene como una de sus características la absorción en la vida diaria de las continuas innovaciones científicas y tecnológicas, y lleva aparejada la demanda de información y la exigencia de comunicación, de diálogo, que posibilite la participación social en la orientación de esos avances.

Un diálogo que se debe construir sobre la base del conocimiento del sentido común porque ese conocimiento "constituye el edificio de significados sin el cual ninguna sociedad podría existir" (Berger y Luckmann, 1986, p. 31).

De ahí la inconveniencia de descartar a aquellos públicos no directamente relacionados con la actividad, ya se trate de empresas o de instituciones, (García, Carrillo y Castillo, 2012; Victoria, 2005; Olvera-Lobo y López-Pérez, 2013). Porque las compañías ya no solo producen, han dejado atrás el título del famoso artículo de Friedman (1970), "La responsabilidad social de las empresas es aumentar sus ganancias", y hoy buscan también incrementar sus relaciones sociales. Las nuevas tendencias de Responsabilidad Social Corporativa (RSC) van en esa línea; y, en la parcela de las biotecnológicas, una de las áreas de mayor trascendencia en las que compartir es, precisamente, la que ellas generan: la del conocimiento; un conocimiento ligado a una actividad que tiene repercusiones en la salud, la alimentación, el medio ambiente, las energías... De tal forma que las actuaciones que promuevan su divulgación, como eje de su RSC, cobra una dimensión inusitada.

\section{LA COMUNICACIÓN DE LA CIENCIA}

Ziman (2003) sostiene que el ser humano tiene una necesidad y capacidad natural de conocer, de tal forma que el conocimiento científico no es más que una respuesta, entre otras, a esa necesidad. Y respecto a ese tipo específico de conocimiento, distingue entre la ciencia académica y una nueva forma de producción de conocimiento, que se ha ido abriendo paso de manera progresiva: la ciencia postacadémica, un híbrido entre la ciencia académica y la ciencia industrial. Esta se caracteriza por la aparición de redes de equipos de trabajo, el aumento de las necesidades de financiación y la exigencia de conseguir aplicaciones técnicas útiles para la sociedad, que incluso puedan influir en el modelo económico de un país. Y la irrupción de este nuevo formato ha incrementado la necesidad de socializar, colectivizar, comunicar, la ciencia.
Durant (1990), por su parte, esgrime tres razones por las que se hace necesaria la divulgación. Una de carácter cultural, otra práctica y una más política. La primera atañe a la ciencia como actividad intelectual cuya adquisición es una característica básica en la civilización occidental moderna. La segunda se refiere a que el impacto de la ciencia y tecnología en nuestra vida cotidiana hace necesario que el consumidor disponga de la información precisa para tomar decisiones fundadas. La tercera, por fin, concierne a que la calidad de una democracia depende de que los ciudadanos tengan una adecuada comprensión de los problemas a resolver, entre los que se incluyen los relativos a cuestiones científicas y tecnológicas.

Esa necesidad de compartir nos lleva a un concepto nuevo y antiguo a la vez -el de bien común-que, atendiendo al conocimiento, coincide con la concepción del bien público o comunal tradicional en que es un recurso compartido, un ecosistema complejo, que necesita de la contribución de todos los elementos que se benefician de él; pero se diferencia en que, en el caso de los recursos extractivos como bosques o pesca, el uso -abuso- de una persona reduce los beneficios disponibles para el resto. En nuestro caso, "cuantas más personas comparten el conocimiento útil, mayor es el bien común" (Hess y Ostrom, 2007, p. 5).

Solo con las perspectivas que aportan esos autores ya se justificaría cualquier trabajo de investigación que se adentre en la comunicación de la ciencia, ya que ellos, además, abren sus objetivos y captan un paisaje enorme en el que, por supuesto, se incluyen las empresas, sea cual fuere su tamaño, como elementos implicados en el desarrollo de un diálogo efectivo en torno a las ciencias experimentales.

Hacer accesible el conocimiento científico al público en general, promover la participación de ese público en la orientación de la investigación, no es, por tanto, una moda pasajera. El diálogo, la comunicación entre los diferentes sistemas -empresas, sociedad, administraciones, organismos de investigación públicos y privados...- que se integran en un ecosistema social es imprescindible para fortalecerlo. Cuestión aparte es cómo medir conocimiento, interés, actitudes, etc. (Muñoz, 2002).

Para este trabajo hacemos nuestra la definición de Comunicación de la Ciencia de Bryant (2003, p. 357) como "los procesos mediante los cuales la cultura científica y su conocimiento se ha incorporado a la cultura común". Un proceso que según Burns, O'Connor y Stocklmayer (2003, p. 194) posibilita el diálogo para 
que "el público en general, los mediadores y los profesionales de las ciencias interactúen unos con otros de manera más eficaz".

La divulgación no es un fenómeno nuevo y ha estado presente a lo largo de la historia; lo que sí ha conseguido un impulso inusitado desde la segunda mitad del siglo pasado es la sistematización de los estudios sobre la propia divulgación.

Bauer, Allum y Miller (2007) hacen un ejercicio de esquematización al proponer tres paradigmas que agrupan los enfoques, el periodo de prevalencia y las correspondientes metodologías con las que se ha abordado la divulgación. Son los que denominan como Alfabetización Científica (1960 en adelante), que se corresponde con el modelo del déficit (Hilgartner, 1990), que contrapone la deficiencia del público a la suficiencia de los científicos, y plantea que las actuaciones para limitar ese déficit deben dirigirse al área de la educación pública; la llamada Comprensión Pública de la Ciencia -a partir de la publicación del informe de la Royal Society en 1985- no se centra tanto en la mediación de conocimientos científicos como en las actitudes hacia ella y en las relaciones entre conocimiento y actitud; y el tercero es el paradigma Ciencia y Sociedad -a partir de 1990-, sobre el que Bauer, Allum y Miller (2007) explican que contempla los déficits de conocimiento del público, la actitud o la confianza, pero también los déficit por parte de las instituciones científicas y tecnológicas y sus representantes expertos.

Sea cual sea el enfoque y la motivación en materia de divulgación, lo cierto es que la ciencia está cada vez más presente en la vida de las personas. De ahí que el interés que despierta se acreciente y que los actores industriales muestren un incipiente interés por ella.

\section{LA EMPRESA Y LA COMUNICACIÓN DE LA CIENCIA}

De forma progresiva la comunidad científica, los gobiernos y la empresa han tomado conciencia de que ciudadanía y medios de comunicación ya no son convidados de piedra en lo que atañe al desarrollo y orientación de la ciencia, sino que su hostilidad puede afectar, restringir o incluso vetar programas de investigación concretos (Miller, Pardo y Niwa, 1998). De forma que ya no solo los organismos públicos, sino también entidades privadas están interesadas en comunicar la ciencia y avanzar, "hacia la participación pública como parte de las relaciones públicas de las empresas" (Bauer, Allum y Miller, 2007, p. 88). Mientras que Burns, O'Connor y StockImayer (2003, p. 199) sostienen que, "la comunicación de la ciencia es un campo importante de la empresa, digna de práctica continua y de investigación".

Quizá se trata de un paso que ha venido dado no tanto por convencimiento interno sino para responder a las expectativas de la ciudadanía, si bien "las prácticas de compromiso y la motivación y la voluntad para llevarlas a cabo dependen de cómo los públicos y las cualidades de conocimiento público son construidos por los propios organismos industriales" (Burningham, Barnett, Carr, Clift, y Wehrmeyer, 2007, p. 23). De tal forma que si hasta ahora los públicos contemplados por las empresas casi se limitaban a clientes, proveedores, empleados y administraciones, ahora deben ampliarse para abarcar también a los medios -como intermediarios con la ciudadanía- y a la sociedad, en especial la de su entorno.

Sin embargo, no parece que este sea un reto conseguido a tenor de algunas claves que se apuntan en el artículo referido de Burningham sobre la industria química británica. Un estudio en el que participaron tanto empresas que producen directamente para el consumidor final (B2C), como otras que venden a otras empresas (B2B), y de distinto tamaño. Resulta especialmente de interés la concepción que sus ejecutivos tienen del público, básicamente, como consumidor. O que "mientras que los encuestados describieron su propio conocimiento, basada en la ciencia 'dura', caracterizan las preocupaciones ambientales 'de los ciudadanos' como esencialmente parcial y egoísta" (Burningham et al., 2007, p. 31).

Igualmente, identificaron junto al interés propio, la cobertura de los medios de comunicación y las ONG como clave en la formación de actitudes y preocupaciones del público, y a esos medios de comunicación como parciales, superficiales y generadores de un miedo injustificado hacia los productos químicos, producto de la influencia de las ONG. Es decir, esos ejecutivos conciben un modelo de transmisión lineal, de las ONG a los medios y al público, al que representan como receptores pasivos de información parcial, sesgada y sensacionalista. Y la respuesta más repetida al requerir a los encuestados por la posibilidad de que sus empresas iniciaran un diálogo con la ciudadanía y suministraran formación e información para aliviar la carencia de conocimiento científico que le achacaban, fue que este tipo de conocimiento es demasiado complejo y los legos no pueden entenderlo.

Otro aspecto de interés es el del crédito que los ciudadanos están dispuestos a depositar en los portavoces o expertos en ciencia, sobre el que llama la atención Wynne (1992, p. 282): 
"se acepta cada vez más que los problemas de comprensión pública de la ciencia, y de la percepción del riesgo, no son tanto acerca de las capacidades públicas en la comprensión de la información técnica, sino de la confianza y la credibilidad que están dispuestos a invertir en portavoces o instituciones científicas".

La cuestión de la confianza y credibilidad no es menor, y parece conveniente que las empresas hicieran un mayor esfuerzo por neutralizar la idea asentada de que la información que transmite un científico que trabaja en un hospital o una universidad es más confiable que el emitido por otro que opera en una empresa privada. Según el estudio internacional de "cultura científica» de la Fundación BBVA, en la media europea de los países estudiados, los médicos, seguidos de los científicos y la comunidad educativa, ocupan los tres primeros puestos como profesionales que más contribuyen al bienestar y avance de la sociedad. De hecho, las puntuaciones obtenidas se elevan para los médicos a 8,2 puntos sobre 10 , y para los científicos a 7,9 puntos; 8,5 y 7,9, respectivamente, en el caso de España. Números que concuerdan con los que arroja la VI Encuesta de Percepción Social de la Ciencia y la Tecnología 2012, según la cual las profesionales más valoradas por los españoles son los médicos, con una puntuación de 4,46 puntos sobre 5 , seguidos de los científicos con 4,24 puntos.

Pero, además, hay que tener en cuenta que el lugar de trabajo no es inocuo. La naturaleza de la institución en la que los científicos desarrollan su actividad introduce matices significativos en el nivel de confianza generado, aun dentro del marco de alto nivel de crédito que la ciudadanía les confiere. De tal forma que en el estudio de la Fundación BBVA, la puntuación media que otorgan los europeos a aquellos que trabajan en hospitales y en las universidades se sitúan a la cabeza, ambos con 7,3 puntos ( 8 y 7,8 , respectivamente, en el caso de España), mientras que los que operan en empresas privadas caen hasta los 5,9 puntos en la media Europea y 6,6 en España, calificación que los sitúa, en el caso nacional, en la última posición de entre las opciones que se les ofrecieron a los encuestados. Por detrás no solo de los que ejercen en hospitales y universidades, sino también, por este orden, en grupos ecologistas, en instituciones de defensa y seguridad, y al mismo nivel que los científicos empleados en órganos y agencias estatales.

Sobre esa cuestión habría que traer a colación algunas ideas sobre las que se incidió en el focus group organizado bajo el amparo del proyecto IBAC 3.5 (Informe de Biotecnología Aplicada a la Comunicacion) que reúne a periodistas, representantes de compañías biotecnológicas y expertos en transferencia tecnológi$\mathrm{ca}$, en el que se proponen claves para mejorar la colaboración entre científicos, empresas y periodistas. En dicha reunión se puso de manifiesto que uno de los problemas que atañen a la divulgación sobre temas biotecnológicos es el ámbito de acceso a las fuentes, y así, se insiste en que "si las empresas o instituciones lanzan una información, deben tener previsto quién va a hablar con los medios; y deben dejar su contacto y que el periodista pueda preguntar sin miedos ni cortapisas" (p. 11). Y es que este tipo de dificultades puede hacer que el periodista desestime informaciones importantes, de manera que llegan menos relatos de carácter científico, de origen empresarial, a los ciudadanos. Debemos tener en cuenta, además, que la accesibilidad puede contribuir a incrementar la sensación de transparencia corporativa.

\section{OBJETO E HIPÓTESIS}

En este trabajo nos centramos en las pequeñas y medianas empresas (pymes) y en una actividad concreta, la biotecnológica, al tratarse de un sector emergente para Europa (An Integrated Industrial Policy for he Globalisation Era Putting Competitiveness and Sustainability at Centre Stage) que el Gobierno europeo incluye, junto a otras, entre las tecnologías facilitadoras esenciales (KET); esto es, unas tecnologías "que son de importancia sistémica para la capacidad de innovación de la industria y del conjunto de la economía" (A European strategy for Key Enabling Technologies - A bridge to growth and jobs, p. 3). Las califica, además, de transversales al entender que su carácter multidisciplinar no solo se traduce en la integración de diferentes tecnologías, sino que pueden traspasar campos y contribuyen a la solución de diferentes retos sociales. Igualmente, el Gobierno español apunta en el mismo sentido (Valero Artola, 2013).

Restringimos el estudio a Andalucía (España), dado que esta Comunidad Autónoma se ha situado de forma consecutiva durante los últimos cuatro años como la región que más empresas biotecnológicas ha creado, acumulado que la sitúa como la tercera región en España por volumen de biotech, al acaparar el 13,5\% de la totalidad nacional, y la segunda en número de empresas usuarias de biotecnología con el 10,89\% (Informe Asebio 2012). Además, hemos optado por limitarnos a aquellas asentadas en los parques tecnológicos andaluces que acogen al menos dos empresas de este tipo: cuatro en total. Se trata de las empresas biotecnológicas ubicadas en los parques de Sevilla -Parque Científi- 
co Tecnológico Cartuja, PCT Cartuja-, Málaga -Parque Tecnológico de Andalucía, PTA-, Granada -Parque Tecnológico Ciencias de la Salud, PTS- y Córdoba -Parque Científico Tecnológico de Córdoba, Rabanales 21-.

En total 32 pymes, dado que hemos descartado a las grandes empresas cuyos recursos para afrontar su comunicación corporativa y su RSC son muy diferentes de las pequeñas; como también lo son las problemáticas que les puedan afectar; además, al tratarse de empresas multinacionales, la política comunicativa se traza en sus sedes centrales, lo que complicaría un estudio como el que planteamos.

El perfil tipo de empresa biotecnológica que se asienta en los parques tecnológicos andaluces es una spin off; micropyme, con menos de 10 empleados y facturación por debajo de los 2 millones de euros; y joven, con una existencia inferior a una década (Martínez-Rodrigo y García-Hernández, 2014).

La hipótesis de la que partimos es que las pymes biotecnológicas no han establecido un diálogo con la sociedad más cercana al entender que su público lo constituyen, casi en exclusiva, sus clientes, no su entorno ni la sociedad en general. Esa ausencia de relación previa anula la posibilidad de integrar mecanismos de divulgación como vía para estrechar lazos con la ciudadanía. El hecho de convivir con otras empresas de base tecnológica (EBT) en recintos muy especializados puede introducir matices que conviene explorar.

\section{METODOLOGÍA}

\section{Los sitios web}

Analizamos los sitios web de 30 de las 32 empresas objeto de estudio, dado que dos de ellas carecían de presencia en Internet al inicio de 2013. Comprobamos la aparición de ese grupo y de los parques que las acogen, en los periódicos locales de referencia en sus respectivas provincias. Y completamos el trabajo con las respuestas que nos han dado 30 directivos de otras tantas empresas biotecnológicas que han contestado la encuesta que les propusimos, tras la imposibilidad de contar con la colaboración de las dos empresas restantes.

En el capítulo de los sitios web hemos utilizado diversas metodologías. Para analizar los textos de la página de inicio una metodología cualitativa de investigación, mediante la técnica del análisis del discurso. Nos hemos servido de unas plantillas de elaboración propia en las que recogemos información acerca de la estructura matriz de cada uno de los discursos encon- trados. Igualmente, mediante el análisis de contenido, comprobamos cómo se presentan las compañías y a qué públicos se dirigen (Victoria Mas y GarcíaHernández, 2013). Hemos compuesto un corpus de ocho sustantivos (abordados como campos semánticos) cuatro de ellos referidos a la actividad de estas empresas: negocio, uso, innovación y tecnicismos; el resto se engloba en sus vinculaciones con el entorno físico y social, no solo el más próximo: sociedad, ciudad -entendida como entorno-, beneficios y persona. En cuanto al resto de las páginas del sitio, para discriminar su utilidad como elemento divulgativo comprobamos la posibilidad de consultar dosieres o informes que permitan ampliar y profundizar sobre informaciones concretas. También, nos detenemos en el análisis de la interactividad de esos sitios, centrada en la presencia en redes sociales: entradas y seguidores. Por último, para comprobar si las empresas analizadas atienden a través de sus webs a los medios como audiencia, dado que representan una vía intermedia para conectarla con la sociedad, hemos seguido de forma esquemática el patrón que utilizaron GonzálezHerrero y Ruiz de Valbuena (2006) en su estudio sobre las relaciones de compañías internacionales con los medios a través de sus webs.

\section{Presencia en periódicos locales}

Para acreditar cuál es la presencia efectiva de las pequeñas y medianas empresas biotecnológicas en los medios nos centraremos en los diarios locales con mayor difusión y mayor número de lectores durante 2012 en las provincias de Granada, Málaga, Sevilla y Córdoba, según los datos de la Oficina de Justificación de la Difusión (OJD) y el Estudio General de Medios (EGM): Ideal, Sur, ABC (edición de Sevilla) y Córdoba. Para analizar la presencia y el tratamiento que los diarios dan a los cuatro parques objeto de estudio y a las empresas biotecnológicas que allí se asientan, hemos recurrido al análisis de contenido de los textos que les dedican en los diarios en papel, por considerarlo idóneo si se aplica con el sentido que le conceden Berelson (1952) y Wimmer y Dominick (1996).

Entre las categorías analizadas, atendemos a las apariciones de empresas y parques en el diario de referencia en la provincia; a las personas que aparecen en esas informaciones, para comprobar si sus gestores son tratados como fuente en temas de ciencia o empresa; y a los temas que abordan esas informaciones, entre otros (García-Hernández, 2014). De ese proceso hemos obtenido un corpus de textos sobre el que hemos trabajado en su to- 
talidad. Todos los resultados, una vez recopilados y codificados, se han trasladado a tablas de Excel para facilitar su manejo, ya que hemos acumulado 365 informaciones.

\section{La opinión de los empresarios}

Para sistematizar la recogida de información y opiniones de altos ejecutivos de las empresas analizadas hemos optado por la utilización de una técnica de carácter cuantitativo, la encuesta. En ella se incluía una treintena de preguntas, tanto dicotómicas, como abiertas y escalas. Una vez hemos dispuesto de las respuestas de cada uno de ellos, se han codificado y se han grabado en hojas de Excel para facilitar la obtención de resultados estadísticos. En concreto, para algunos bloques de datos nos ha resultado suficiente extraer porcentajes, en especial para aquellos resultados en los que les habíamos ofrecido a las empresas opciones de respuesta dicotómicas. En cuanto a las contestaciones que se ampliaban con escalas de 10 puntos optamos por extraer medias y desviación estándar para la obtención del respectivo margen de error.

\section{RESULTADOS}

\section{Los sitios web}

La mayoría de las empresas optan en los textos de la página de inicio de sus sitios web por una estructura expositiva en la que combinan las referencias al quiénes son con el qué hacen (2/3 partes del total), mientras que $1 / 4$ se limita a exponer qué hacen. $Y$ solo tres de ellas (10\%) alude, aunque tangencialmente, a las consecuencias no socioeconómicas (calidad de vida y medioambiental) que sus actividades implican. De tal forma que los códigos que utilizan son meramente expositivos y se ha descartado la oportunidad de aportar interpretaciones (hermenéutica) sobre su actividad y sobre la propia empresa. No encontramos contribución, a través de esos sitios, a ampliar las competencias de los lectores que se acerquen a ellos, posibilidad que según Eco (1981) tiene todo tipo de texto.

En cuanto al análisis de contenido, hay que matizar en lo relativo a la presencia de tecnicismos, presentes en el $90 \%$ de las páginas de inicio, que tienen una alta variabilidad; desde aquellas páginas -en especial unaen la que este tipo de voces aparecen encadenadas hasta convertir el texto en jerga, hasta aquellas en las que su presencia es casi testimonial y, aunque se trate de palabras propias de su técnica, su uso es habitual en registros menos formales. Salvada esta aclaración, los resultados nos ofrecen una clara separación entre los dos bloques propuestos. Frente a una presencia, superior al $80 \%$ en todos los casos, de los campos semánticos de las cuatro palabras seleccionadas que se centraban en la actividad de las empresas, se da una presencia casi testimonial -inferior al 20\%-de aquellos sustantivos que engloban las vinculaciones con su entorno físico y social. La escasez de términos relativos a la "persona" es especialmente significativa: solo el $20 \%$ los contempla, pero en casi todos los casos las referencias son a conceptos genéricos, tales como "investigación y tecnología aplicada al bienestar del hombre" o "mejora de la calidad de vida", lo que implica un cierto distanciamiento que no parece casual. Así, en varios textos se hace referencia a enfermedades, pero no a las personas que los padecen $y$, en los casos en que se alude a ellas, aparecen con la palabra "paciente".

La excepción en ese bloque llega de la mano de "beneficios", presente en la mitad de las páginas analizadas, pero en ellas se alude al tratamiento de enfermedades, mejora de la salud, aprovechamiento de residuos, sin profundizar en ninguno de ellos y sin alusión alguna a otros provechos que su actividad genera para la sociedad en general y su entorno en particular sin referencias a los individuos que se beneficiarán de sus productos, servicios o procesos (Gráfico 1).

Esa parquedad relacional se completa con la escasez de dosieres o informes, presentes solo en el $35 \%$ de los sitios analizados, que carecen de un enfoque divulgativo.

En lo relativo a la interactividad encontramos que el $40 \%$ de los sitios disponen de enlaces a una red social al menos. De entre ellos el más habitual es Twitter, presente en el 33\% de los sitios; seguido de Facebook, el $30 \%$; Linkedin, el $26 \%$ y YouTube, el $13 \%$. Además, las actualizaciones escasean y solo la mitad de ellas se pueden considerar activas en alguna de las redes en las que tienen presencia.

El espacio que podríamos considerar como sala de prensa virtual no existe como tal en ninguno de los sitios analizados; lo más parecido es un cajón de sastre bajo el epígrafe Noticias, dispuesto en el $80 \%$ de los casos. En él se recogen notas de prensa, varias sin datar; apariciones en medios, algunas con link muertos; lanzamientos de productos; acuerdos comerciales; etc. Ninguna dispone de imágenes para descargar, ni recursos multimedia, cuyo acceso se dispone a través de YouTube en los sitios en que los ofrecen. 
Gráfico 1. Campos semánticos en página de inicio

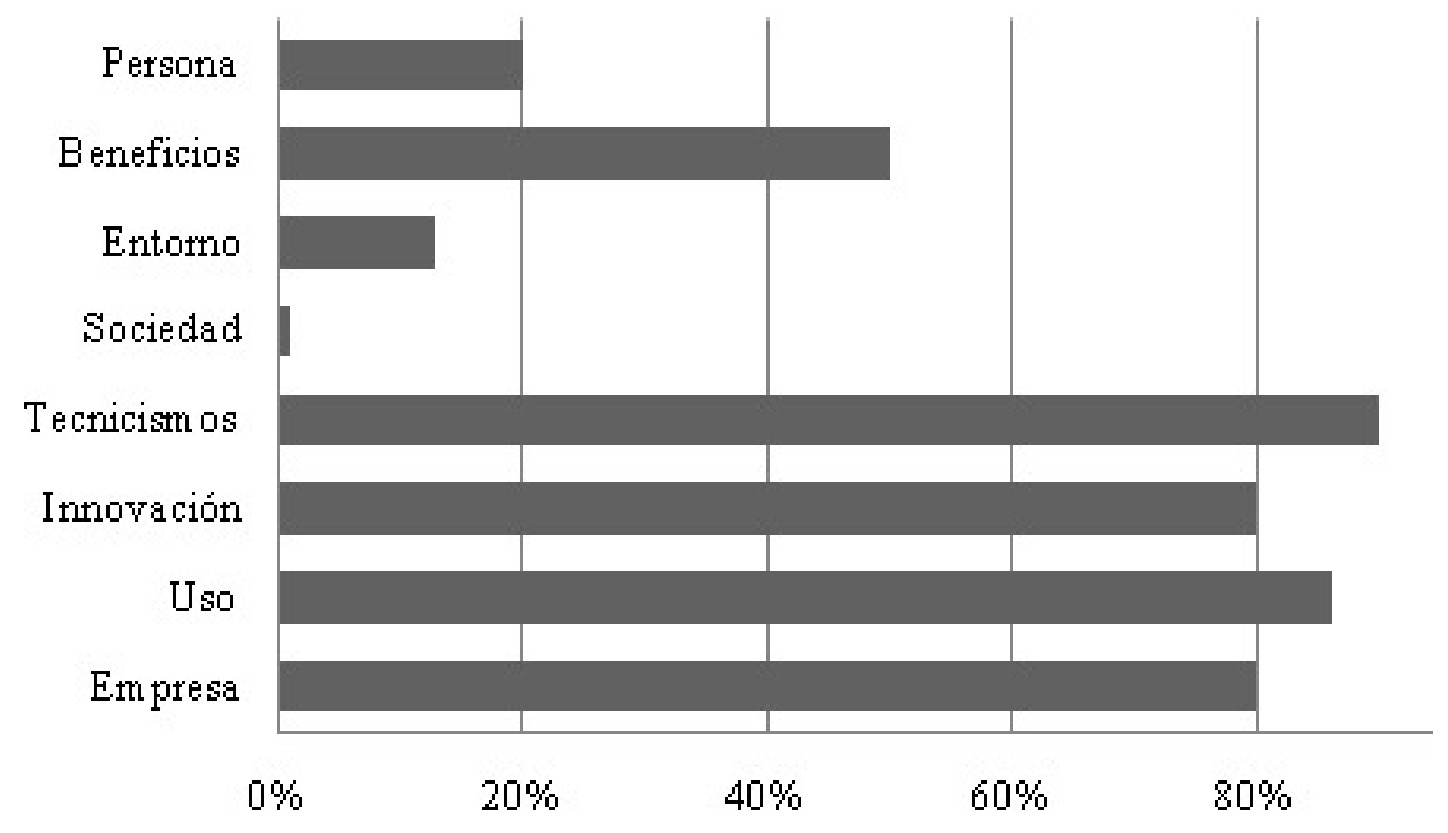

Fuente: Elaboración propia.

Entendemos que esos resultados avalan la apreciación de que los argumentos y estructuras sobre los que se asientan los textos de los sitios web analizados no se han elegido de forma casual, sino que están al servicio de llegar a unos lectores que poseen las competencias y experiencias que les permiten descifrarlos (Van Dijk, 1990), y se ha descartado llegar a otros. De ahí que, además de obviar el para qué, para quiénes y cómo hacen lo que hacen, no se incluyan en estos sitios alusiones a las repercusiones socioeconómicas de su actividad, espacios específicos para aportar información que contribuya a crear competencias en las personas que se acerquen a esas páginas y los mecanismos de diálogo sean escasos. Pareciera que estas empresas todavía no son conscientes de que internet se ha convertido para los españoles en la primera fuente de información científica (Informe de la FECYT sobre "percepción social de la Ciencia y la Tecnología 2012).

\section{Presencia en periódicos locales}

Cuando nos centramos en la presencia en los periódicos de referencia de cada una de las provincias donde se asientan los parques, encontramos en 2012 un total de 101 textos en la edición de Granada del diario Ideal, en los que se aludía al Parque Tecnológico de Ciencias de la Salud, o a alguna de las empresas allí instaladas (Gráfico 2).
Del total de esas referencias, el $8 \%$ tienen como eje a alguna de las empresas objeto de estudio. En el $75 \%$ de las ocasiones predominan los elementos empresariales, mientras que el ámbito biotecnológico está presente en la mitad de los textos. Debemos resaltar que las declaraciones de sus gestores se limitan a estas páginas, sin que se registre su presencia como expertos en otras informaciones.

El siguiente bloque lo constituyen las 93 informaciones restantes en las que se menciona al PTS. En ese paquete se engloban diez informaciones en las que, simplemente, se nombra a alguna de las empresas biotecnológicas asentadas en el PTS, aunque los escritos abordan temas distintos a su actividad investigadora o empresarial.

Especial relevancia tiene el tema que abordan esos textos, ya que en el $69 \%$ de ellos aparece la política como marco y el PTS como elemento de confrontación partidista. En cuanto a las actividades, incluidos los foros y acuerdos, en los que ha participado la Fundación Parque Tecnológico de la Salud, le corresponden el $10,7 \%$ de las informaciones registradas en 2012 por el diario Ideal. Se trataba de acuerdos de colaboración financiera con entidades crediticias granadinas, visitas institucionales y participación en eventos. Los temas relacionados con la economía en 
Gráfico 2. Referencias en 2012

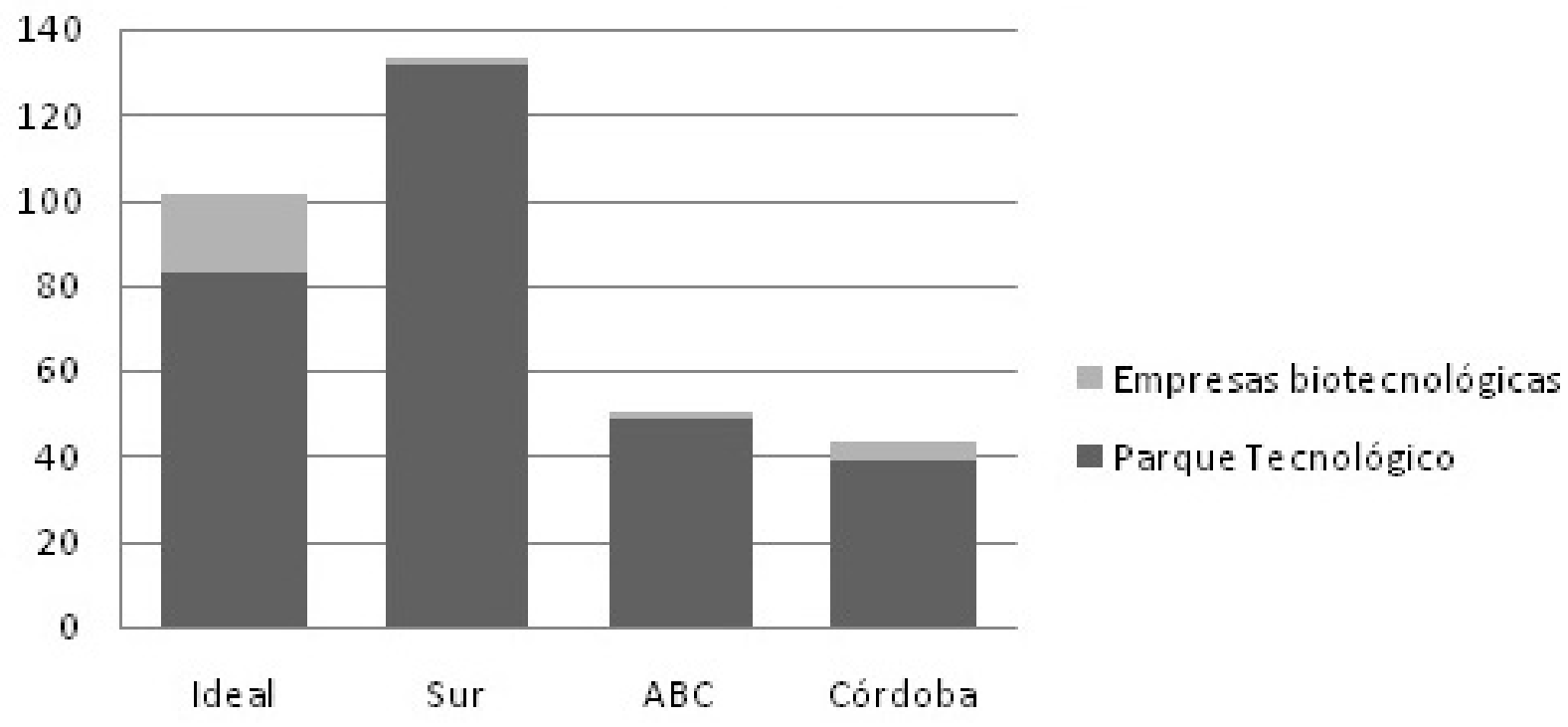

Fuente: Elaboración propia.

los relatos del periódico acerca del PTS, aparecen en el $12,9 \%$. Algo menos, el $11,8 \%$, representan los elementos propios de la divulgación, pero siempre aparecen en confluencia con otros temas y se limitan al ámbito de lo que Escribano y Quintanilla (2005, p. 27) denominan "política científica" (Gráfico 3).

Un total de 172 alusiones (133 de ellas en la edición en papel) al PTA, o a alguna de sus empresas en el diario Sur de Málaga, dan cuenta de que este parque se ha hecho un hueco en las páginas del diario, de forma que en 2012 se asomó una media de algo más de 2,5 veces a la semana. Los nombres de alguna de las empresas del parque figuran en 55 ocasiones, y solo en una de ellas aparece el de una biotecnológica, pero sin relevancia en la información y sin aparición de sus gestores, ni en esa ni en otra información.

Gráfico 3. Elementos divulgativos (incluidos de política científica)

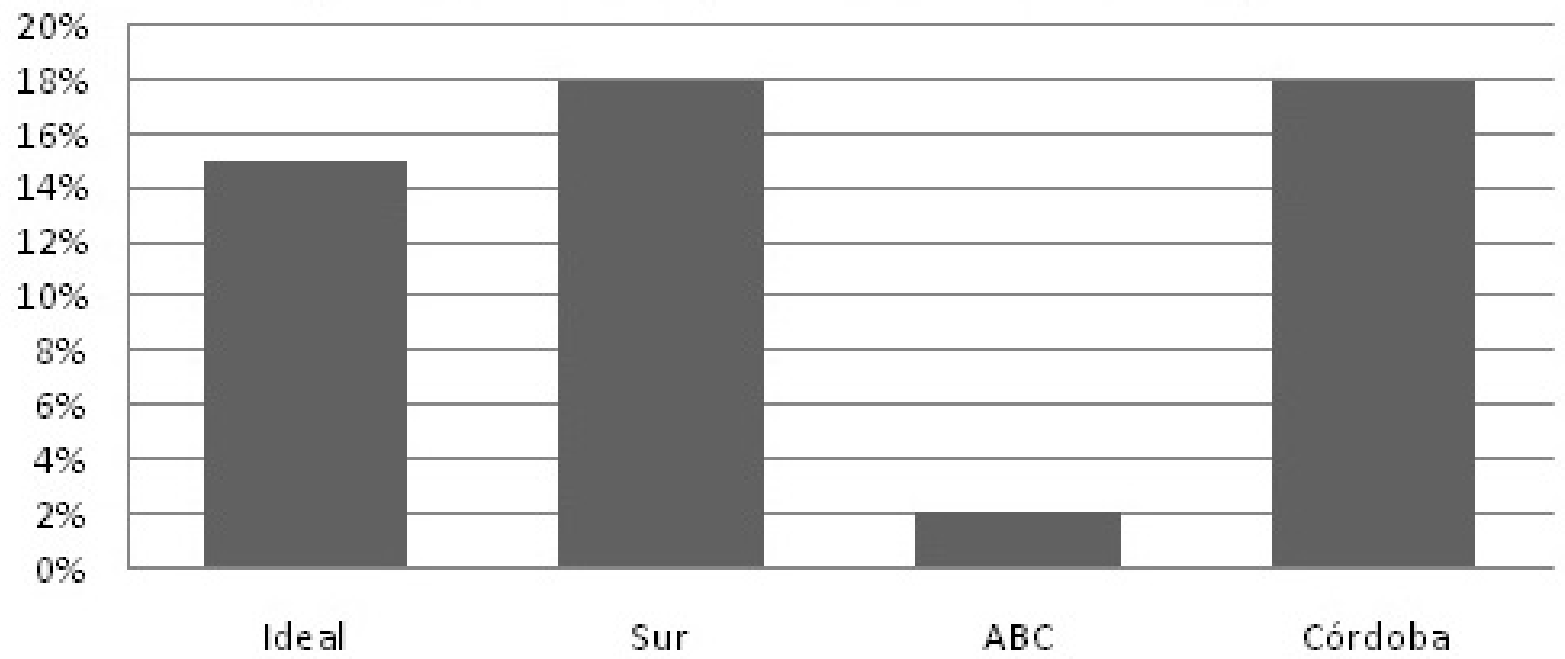

Fuente: Elaboración propia. 
En el análisis de los temas abordados en los textos, destacan los marcos económicos y empresariales, en el $62 \%$ de los casos. La innovación está presente en el $27 \%$ de ellos. Mientras que elementos vinculados a la divulgación figuran en el $18 \%$, aunque en varias ocasiones se plasma un texto técnico, sin claves divulgativas, $y$, además, se vuelcan en el campo de la política científica más que en la divulgación, que solo aparece en dos de esos textos. Por su parte, las referencias políticas están presentes en el $18 \%$ de las informaciones, si bien esos temas no suelen ser el eje de las informaciones analizadas, sino que su carácter es más tangencial. Y mención aparte merecen las alusiones a actos y eventos organizados por el PTA o en los que el parque colabora, ya que en el $34 \%$ de las ocasiones es el contexto que enmarca el relato periodístico.

En total 49 informaciones sobre el Parque Científico Tecnológico Cartuja, ubicado en Sevilla, se reparten a lo largo del año 2012 por las páginas del diario $A B C$, en su edición sevillana. Se registran ocho informaciones en las que se nombra a empresas del recinto (16\%), y solo en una de ellas se nombra a una empresa biotecnológica, sobre la que se hace una leve referencia a su actividad. No figuran declaraciones de los gestores en ese ni en ningún otro texto del diario. En el bloque de informaciones en las que se hace alusión al PCT Cartuja destaca que 17 de ellas, el 34\%, están directamente relacionadas con el veinte aniversario de la Expo 92, y las referencias al Parque se limitan a recordar la coincidencia de ubicación. En cuanto a los temas a los que se alude, además del mencionado de la Expo, sobresale el económico, presente en el $40 \%$ de los relatos; las alusiones a infraestructuras y equipamientos están en el 32\% de los textos; y aspectos políticos aparecen en el 36\% de las ocasiones. Queremos destacar, igualmente, lo que no hallamos en las páginas analizadas, ni mención a la innovación, ni alusiones a la presencia del Parque como organizador o participante en eventos de carácter empresarial o divulgativo, convenios de colaboración, etc. Solo en uno de los textos se hace referencia a política científica con la apertura de un edificio en el PCT Cartuja destinado a la divulgación.

El diario Córdoba hace a lo largo del ejercicio 2012 un total de 43 referencias al Parque Tecnológico Rabanales 21, en cuatro de las cuales se menciona expresamente alguna de las empresas biotecnológicas allí asentadas. Dos de esas informaciones se refieren a los edificios que las albergan y apenas se da cuenta de la actividad empresarial que desarrollan. Algo más extensos y con mayor profundidad son los tratamientos que se otorgan a las otras dos informaciones, en una de las cuales se recogen declaraciones del director general de una biotecnológica. Pero no se registra a lo largo del año ninguna otra declaración de los gestores de alguna de las biotecnológicas asentadas en Rabanales 21.

En el diario Córdoba la distorsión en el recuento viene de la mano del encierro en la incubadora de empresas de los empresarios que la habían construido, por un impago. La atención a ese conflicto representa más del $25 \%$ del total de informaciones del recinto. Cabe mencionar que la presencia de temas políticos en asuntos ligados al Parque se queda en el 30\%, porcentaje superado por aquellas informaciones que giran sobre actividades desarrolladas o participadas por Rabanales 21 -el 32,5\%-. Por su parte, las informaciones que tocan, aunque no sea el eje de esas informaciones, implicaciones económicas o empresariales del recinto alcanzan el $32 \%$; si el tema es la innovación, sola o ligada a otros, nos situamos en el $11,6 \%$, y la presencia de elementos divulgativos está en el 18\%. Sin embargo, en este último grupo predominan las piezas de lo que denominamos política científica, casi el $10 \%$.

Como corolario de este segundo bloque de análisis podemos apuntar que las empresas biotecnológicas, que conforman nuestro objeto de estudio, se cuelan tan poco en las páginas de los periódicos locales, que no pueden contribuir a asentar la idea de que estamos ante empresas valiosas, para su entorno y para la sociedad en general. Pero esa falta de conexión se agrava si los propios parques tienen una presencia ridícula en esos medios, con la excepción del PTA. En el caso de Rabanales 21, su escasa aparición en el diario Córdoba parece justificada, ya que el primer vivero de empresas consiguió los permisos municipales de ocupación en diciembre de 2011. Sin embargo, esa coartada no existe para el PTS, cuyo nombre asoma por las páginas del periódico Ideal, en demasiadas ocasiones, ligado a la confrontación política; ni para el PCT Cartuja, por su asociación a la pasada EXPO 92. Además, la existencia de vínculos de los parques con su entorno a través de la participación en actividades, de diversa índole, de las que se hicieran eco los periódicos es de tal pobreza que merecería una reflexión de responsables empresariales y gestores de los parques.

\section{La opinión de los empresarios}

El tercer bloque de este análisis lo constituyen las respuestas de los ejecutivos de las pymes objeto de estudio. El 30\% de las empresas consultadas ha ejecutado alguna acción comunicativa a través de medios tradicionales en 2012 , en todos los casos se tra- 
taba de lanzar notas de prensa con un resultado que estiman positivo menos de la mitad (44\%) de los que desarrollaron tales ejercicios. En cuanto a acciones de comunicación externa más complejas, tales como jornadas de puertas abiertas, contactos con colegios de su zona, conferencias para no expertos en materia biotecnológica, participación en foros vecinales... La respuesta positiva es escueta, tan solo tres de las encuestadas (10\%) habían participado en eventos de este tipo en el año de referencia. Hay que destacar, igualmente, que solo el $53 \%$ de los encuestados considera valiosa la aportación de un experto para gestionar la comunicación de su empresa.

El 93\% estima que una alianza estratégica, entre las empresas biotecnológicas de los parques respectivos, en asuntos de comunicación externa, podría repercutir en la valoración del recinto como foco de innovación. El 90\% declara que su empresa atesora aspectos que pueden resultar de interés para el ciudadano medio de su ciudad y provincia en materia de empleo, innovación y desarrollo, progreso,... Y el 97\% aprecia un gran desconocimiento entre la ciudadanía de su localidad tanto acerca de la actividad biotecnológica que se realiza en los parques respectivos, como sobre las empresas que la desarrollan; porcentaje que desciende algo, hasta el $70 \%$, cuando se les requiere su opinión sobre si esa falta de ligazón con su entorno lastra el desarrollo empresarial del Parque.
También les hemos solicitado una valoración sobre la trascendencia que le conceden a que los ciudadanos de su provincia conozcan lo que hacen y cómo y para qué lo hacen, a través de los medios locales; la media de las puntuaciones obtenidas asciende casi al notable con $6,7 \pm 0,41$ puntos sobre 10 . Si la pregunta gira en torno a los medios de carácter económico, la calificación se eleva hasta los 7,16 $\pm 0,48$ puntos; mientras que si la evaluación se refiere a la importancia que consideran que tiene para su empresa la publicación de artículos en revistas científicas, la nota se adentra en el notable alto con 8,1+0,36 puntos de media (Gráfico 4).

Además, hemos comprobado que esas diferencias de resultados son estadísticamente representativas. Al verificar dónde se concentran esas divergencias, mediante el test de Student Newman-Keuls, encontramos que se hallan entre los resultados correspondientes a las valoraciones de las revistas científicas respecto a los medios económicos y los generalistas locales, pero no entre medios económicos y generalistas locales, que arrojan un valor de $\mathrm{P}>0,05$, por lo que no podemos concluir que entre estos últimos las diferencias no procedan del azar. Una característica esta que puede derivarse de su origen, mayoritariamente universitario, en donde las publicaciones científicas tienen un gran peso específico para evaluar la capacidad investigadora.

Gráfico 4. Valoración de medios

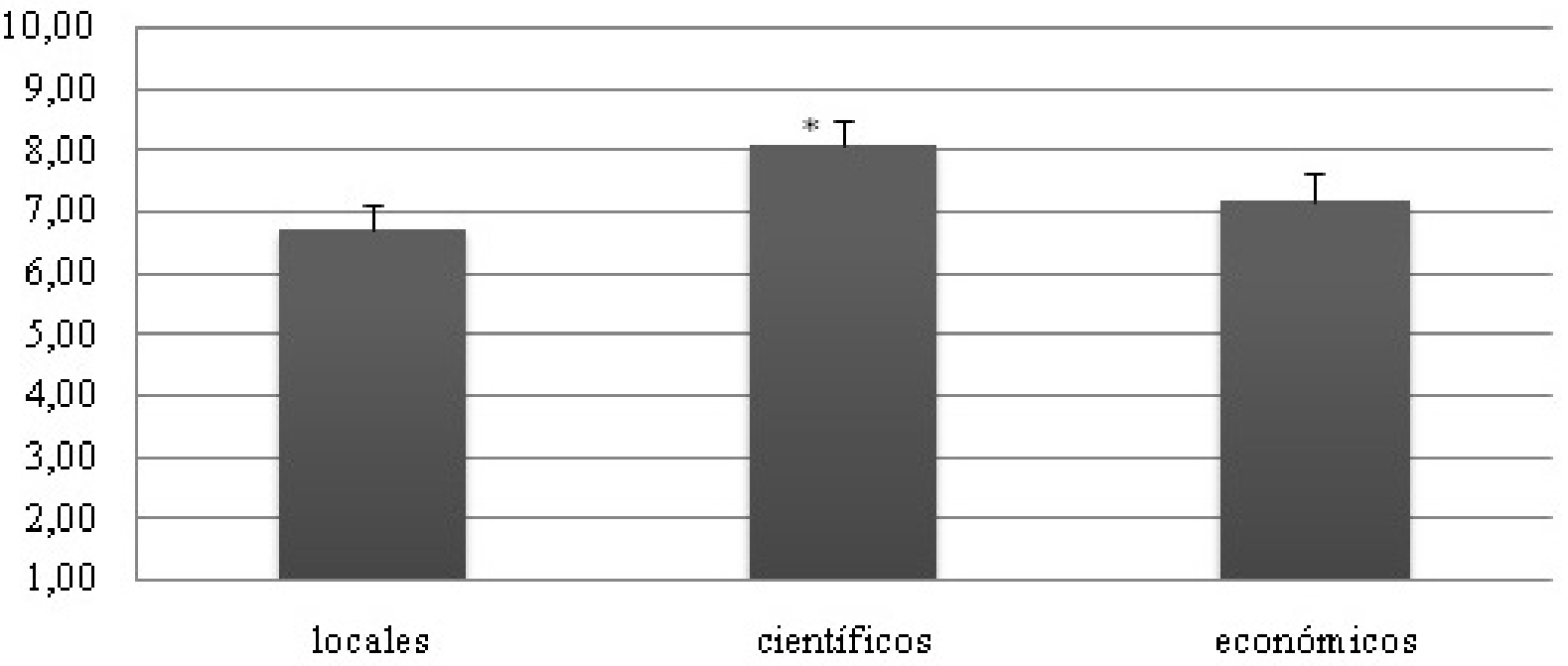

Fuente: Elaboración propia. 
Por último, requerimos a los ejecutivos que respondían al cuestionario para que valoraran una apreciación de Grunig y Hunt (2003, p. 401) según la cual:

\footnotetext{
"Muchas organizaciones se involucran en sus comunidades locales a causa de una sensación de responsabilidad social y no porque crean que su propio bienestar está involucrado... Sin embargo, la mayoría de las organizaciones participa en la comunidad porque se da cuenta de que una comunidad fuerte le ayuda a ser una organización más fuerte".
}

Algunas de las empresas (37\%) no comentaron la cita o se limitaron a un "no sé" o "puede ser", pero el $23 \%$ mostró su desacuerdo con tal apreciación con respuestas que van desde, "nuestra demanda está fuera" o "no dependemos de la idea que la ciudad tenga de nosotros", hasta "estamos en este parque andaluz porque las ayudas públicas recibidas nos obligan, pero no tenemos vinculación con la comunidad local, ni la consideramos necesaria". Por su parte, las declaraciones positivas alcanzan al $40 \%$ de las respuestas, aunque algunos ejecutivos, dentro del acuerdo hacia la referencia mostrada, también insisten en que sus clientes no son locales.

De la información que nos han proporcionado las encuestas, extraemos una serie de planteamientos que quizá deriven directamente del tamaño de estas empresas y su disponibilidad de recursos; es el caso de que casi la totalidad considere que la colaboración en materia de comunicación, entre las empresas de su sector asentadas en el parque, sería una buena forma de acercarse al exterior. No sería más que una extensión de otras colaboraciones a las que están habituadas al participar en programas de $I+D+i$ autonómicos, españoles o de la UE (Holl y Rama, 2012). Sin embargo, cuando atendemos a la praxis, esta es limitada y destaca que solo tres de esas empresas hayan acometido acciones directas de comunicación externa, sin la intervención de los medios de comunicación. Destacamos, igualmente, la contradicción que aparece en dos resultados. Por un lado, la cita de Grunig, que sugiere que su propio futuro está ligado al de su comunidad, es compartida por menos de la mitad de los encuestados; aun cuando algo antes, al completar el cuestionario, el $70 \%$ había sostenido que la escasa ligazón entre la ciudadanía de su localidad y las empresas lastraba el desarrollo empresarial del Parque. El matiz, entendemos, está en que cuando el entrevistado capta la pregunta desde una posición más general -sector de las biotecnológicas o Parque-, la respuesta es una, y cuando la centra sobre la empresa propia, es otra.

\section{CONCLUSIONES}

Las empresas biotecnológicas analizadas no utilizan sus sitios web como lugares donde encontrarse con la ciudadanía, dialogar con ella, presentarse como parte de la sociedad o abrir su sector y contestar las preguntas tácitas que los no expertos se hacen acerca de una actividad tan nueva y, a la vez, tan antigua como la producción del pan o el vino. Esa falta de perspectiva limita las opciones de que aprovechen todas las oportunidades que ofrece internet, por apuntar exclusivamente en los clientes reales o potenciales, olvidando que especialmente la población más cercana puede ser una aliada, si dan a conocer su existencia, porque lo que no se conoce es como si no existiera.

En cuanto a la presencia de las empresas biotecnológicas andaluzas y los parques que las acogen en los periódicos locales de referencia, en sus respectivas provincias, buscábamos conocer si las pymes biotecnológicas asentadas en parques tecnológicos andaluces han abierto una vía de comunicación con su entorno, a través de las páginas de esos periódicos, y la respuesta es negativa; si sus gestores, normalmente científicos en los casos que nos ocupan, se han convertido en fuentes recurrentes al abordar temas relacionados con la biotecnología, y la respuesta es también negativa; si las empresas de los parques son cauce por el que transita la comunicación de la ciencia a través de los medios más cercanos, y la respuesta es, de nuevo, negativa.

No contamos con suficientes datos objetivos como para saber si esa falta de relación entre medios locales y empresas de los parques es responsabilidad de las propias empresas, tan absortas en su cotidianeidad como para atender a aquello que las relacione con el exterior; o de los periódicos, a los cuales les resulta más fácil llenar sus páginas locales asistiendo a ruedas de prensa que buscando información en donde se produce la novedad. Lo que sí es achacable a los medios de comunicación es la escasa presencia de elementos de divulgación más allá de los suplementos específicos de innovación; este encorsetamiento no se produce, por ejemplo, con la economía, que ha saltado de su sección para impregnar al resto.

Por lo que se refiere a la información que nos han proporcionado los cuestionarios, debemos destacar la buena disposición que muestran los encuestados hacia la ciudadanía como público de interés. Elementos que dan cuenta de ello son que entienden que sus empresas reúnen elementos que pueden resultar atractivos y ser valoradas por la población más cercana, que 
valoren a los medios como elemento de acercamiento a la sociedad, o que consideren que sus vecinos no las conocen y esa desconexión puede lastrar el desarrollo del parque tecnológico en el que se asientan. El problema está en cómo solucionar tal separación si solo la mitad de los ejecutivos consultados estima valiosa la aportación de un experto para diseñar y ejecutar la política de comunicación de su empresa.

Abrir los sitios web, tener presencia y actividad en redes sociales, acercarse a los medios, son elementos importantes en la comunicación de las empresas, en especial en las que hemos abordado que desarrollan actividades muy especializadas, aunque con gran incidencia en la mejora de la calidad de vida de las personas, como son alimentación, agricultura, energía, salud... Pero con ser actuaciones importantes, en gran medida descuidadas por las pymes que conformaban nuestro objeto de estudio, no son suficientes para que realmente exista un verdadero diálogo entre ellas y de ellas con la sociedad en general y sus vecinos en particular; el contacto directo, la implicación en la vida de los ciudadanos debería ser más estrecha para poder construir, entre todos, ecosistemas fuertes. Y entendemos que, para estas empresas, y en general para todas las biotecnológicas, integrar la divulgación como el anclaje en el que enganchar la construcción de una comunicación externa fuerte beneficiaría tanto a las compañías como a la sociedad.

Creemos que ha quedado demostrada la hipótesis de la que partíamos; si bien, la ausencia de relación entre sistemas no forma parte de una voluntad aislacionista de las empresas -que sí están interesadas en abrir cauces de diálogo-, sino más bien de una falta de organización del grupo, ausencia de guía -en la mayoría de los casos- y lagunas en la aplicación de procedimientos efectivos.

\section{BIBLIOGRAFÍA}

Bauer, M. W., Allum, N. y Miller, S. (2007). What can we learn from 25 years of PUS survey research? Liberating and expanding the agenda. Public Understanding of Science, 16, 1, pp. 79-95. http://dx.doi.org/10.1177/0963662506071287

Berelson, B. (1952). Content analysis in communication research. New York: The Free Press.

Berger, P. L. y Luckmann, T. (1986). La construcción social de la realidad. Buenos Aires: Amorrortu.

Bryant, C. (2003). Does Australia need a more effective policy of science communication? International Journal for $\mathrm{Pa}$ rasitology, 33, 4, pp. 357-61. http://dx.doi.org/10.1016/ S00207519(03)000043

Burningham, K., Barnett, J., Carr, A., Clift, R. y Wehrmeyer, W. (2007). Industrial constructions of publics and public knowledge: A qualitative investigation of practice in the UK chemicals industry. Public Understanding of Science, 16, 1, pp. 23-43. http://dx.doi.org/10.1177/0963662506071285

Burns, T. W., O'Connor, D. J. y Stocklmayer, S. M. (2003). Science communication: a contemporary definition. Public Understanding of Science, 12, 2, pp. 183-202. http://dx.doi. org/10.1177/09636625030122004

Durant, J. R. (1990). Copernicus and Conan Doyle: or, why should we care about the public understanding of science? Science and Public Affairs, 5, 1, pp. 7-22.

Eco, U. (1981). Lector in fabula. La cooperación interpretativa en el texto narrativo. Barcelona: Lumen.

Escribano, M. y Quintanilla, M. A. (2005). La biotecnología y los medios de comunicación en España. Revista de Ciencia, Tecnología y Sociedad, 4, 2, pp. 21-39.
Friedman, M. (1970, 13 de septiembre).The Social Responsibility of Business is to Increase Its Profits. The New York Times Magazine, pp 32-126. Disponible en: http://graphics8.nytimes.com/ packages/pdf/business/miltonfriedman1970.pdf

García-Hernández, M. L. (2014). Presencia de las pymes biotecnológicas y los parques tecnológicos andaluces en los periódicos locales. En Camarero Calandria, E. (coord.) Contenidos y formas en la vanguardia universitaria. Madrid: $\mathrm{ACCl}, \mathrm{pp}$. 141-158.

García, M., Carrillo, M. V. y Castillo, A. (2012). La usabilidad en los portales web de las pymes. Herramientas y usos para la comunicación empresarial. En: Estudios sobre el Mensaje Periodístico, 18, 1, pp. 365-375.

González Herrero, A. y Ruiz de Valbuena, M. (2006). Trends in online media relations: Web-based corporate press rooms in leading international companies. Public Relations Review, 32, 3, pp. 267-275. http://dx.doi.org/10.1016/j.pubrev.2006.05.003

Grunig, J. E. y Hunt, T. (2003). Dirección de Relaciones Públicas. Barcelona: Gestión 2000.

Hess, C. y Ostrom, E. (2007). Understanding Knowledge as a Commons. From Theory to Practice. Cambridge, MA: MIT Press.

Hilgartner, S. (1990). The dominant view of popularization: Conceptual problems, political uses. Social Studies of Science, 20, 3, pp. 519-539. http://dx.doi.org/10.1177/030631290020003006

Holl, A. y Rama, R. (2012). Technology sourcing: Are biotechnology firms different? An exploratory study of the Spanish case. Science and Public Policy, 39, 3, pp. 304-317. http://dx.doi. org/10.1093/scipol/scs007

Martínez-Rodrigo, E. y García-Hernández, M. L. (2014). Las pymes biotecnológicas y la ciudadanía. Un ecosistema por construir. En 
Gonzálvez, J. E. y Valderrama, M. (coords.) Comunicación actual: Redes sociales y lo 2.0 y 3.0. Madrid: McGraw Hill, pp. 361-371.

Miller, J. D., Pardo, R. y Niwa, F. (1998). Percepciones del público ante la ciencia y la tecnología: Estudio comparativo de la Unión Europea, Estados Unidos, Japón y Canadá. Bilbao: Fundación BBVA.

Muñoz, E. (2002). La cultura científica, la percepción pública y el caso de la biotecnología. Grupo de Ciencia, Tecnología y Sociedad, CSIC. Documento de Trabajo 02-07. Disponible en: http:// digital.csic.es/bitstream/10261/1503/1/dt-0207.pdf

Olvera-Lobo, M. D. y López-Pérez, L. (2013). La divulgación de la ciencia española en la Web 2.0: el caso del Consejo Superior de Investigaciones Científicas en Andalucía y Cataluña. Revista Mediterránea de Comunicación, 4, 1, pp. 169-191. http://dx.doi. org/10.14198/MEDCOM2013.4.1.08

Valero Artola, L. (2013). La política industrial del Ministerio de Industria, Energía y Turismo. Reindustrialización, competitividad y creación de empleo. Economía Industrial, 387, pp. 49-54.

Van Dijk, T. A. (1990). La noticia como discurso. Comprensión, estructura y producción de la información. Barcelona: Paidós Comunicación.

Victoria, J. S. (2005). Reestructuras del sistema publicitario. Barcelona: Ariel Comunicación.

Victoria Mas, J. S. y García-Hernández, M. L. (2013). Las pymes biotecnológicas y su comunicación en la web. En Duran Medina, J. F. Comunicación 2.0 y 3.0. Madrid: Visión Libros, pp. 437-458.

Wimmer, R. D. y Dominick, J. R. (1996). La investigación científica en los medios de comunicación. Una introducción a sus métodos. Barcelona: Bosch.

Wynne, B. (1992). Misunderstood misunderstanding: social identities and public uptake of science.
Public Understanding of Science, 1, 3, pp. 281-304. http://dx.doi. org/10.1088/09636625/1/3/004

Ziman, J. (2003). ¿Qué es la ciencia? Madrid: Cambridge University Press.

\section{Recursos de Internet}

Estudio internacional de "cultura científica» de la Fundación BBVA. Actitudes hacia la ciencia. [En línea] Disponible en: http://www. fbbva.es/TLFU/dat/presentacionciencia.pdf

European Commission. A European strategy for Key Enabling Technologies - $A$ bridge to growth and jobs. [En línea]. Disponible en: http://www.kowi.de/de/Portaldata/2/Resources/fp/2012com-ket.pdf

European Commission. An Integrated Industrial Policy for the Globalisation Era Putting Competitiveness and Sustainability at Centre Stage. [En línea] Disponible en: http://ec.europa.eu/enterprise/policies/industrial-competitiveness/industrial-policy/ files/communication_on_industrial_policy_en.pdf

Informe Asebio 2012. Situación y tendencias del sector de la biotecnología en España. [En línea]. Disponible en: http://www.asebio.com/es/documents/Asebio_2012_web_001.pdf

Informe de Biotecnología Aplicada a la Comunicación (IBAC 3.5). [En línea]. Disponible en: http://www.mercadosbiotecnologicos.com/ documents/informe_zeltia_sobre_medios_comunicacion.pdf

Informe de la FECYT sobre "percepción social de la Ciencia y la Tecnología 2012". [En línea]. Disponible en: http://www.fecyt.es/ fecyt/docs/tmp/363174605.pdf

The public understanding of science. The Royal Society 1985. [En línea]. Disponible en: https://royalsociety.org/ /media/Royal_ Society_Content/policy/publications/1985/10700.pdf 